\title{
Brainwashing, Honeybee Style
}

\section{Giovanni Galizia}

$\mathrm{I}$ n the 1932 novel Brave New World, Aldous Huxley created a society where fetuses develop in bottles and are treated with chemicals to modify their bodies and mentalities (1). Later, children are sleep-conditioned to their future task in society. This procedure creates people who have clear roles, putting them in castes, ranging from alphas (the leaders) to epsilons (the drones). Among other things, lower castes are programmed not to be aggressive against higher caste members. A treatment with neurotoxic chemicals (including alcohol) during development leads to the appropriate brain changes. On page 384 of this issue, Vergoz et al. (2) elucidate some of the chemical cues that influence learning and development in an actual animal caste system - the honeybees.

Within the animal kingdom, social insects have evolved the most stable caste societies. Many ant species have a wide range of castes, from workers to foragers, from groomers to soldiers (3). Individuals all develop from eggs laid by the same mother - the colony's queen. Generally, the food supplied to each egg is the biological signal that leads the embryo to develop into one caste or another, a situation reminiscent of Huxley's fictional world. Thus, if we take as an example the leaf-cutter ant Atta texana, small individuals tend to the fun- gus garden within the nest, intermediate-sized individuals search and collect leaves from the forest to feed the fungus, and large individuals with strong mandibles defend the colony.

Honeybees have evolved a different caste system: The individual worker bees (always females, because male drones do not contribute to social life apart from mating with queens during nuptial flights) perform different tasks in the course of their lives. The workers start off as nurses tending to the hive right after emergence, continue with tasks such as nest-building and hive defense, and then end their lives as foragers by collecting nectar and pollen to make honey and feed their sisters.

How is this developmental polyethism organized? Age is the main factor that determines the task that an individual will perform, mediated by regulation with juvenile hormone (4), but feedback from the hive is also important. Much information is delivered by pheromones. For example, when a hive loses the queen, her queen mandibular pheromone (QMP) will also disappear, leading to rapid changes in behavior among the worker bees, who start raising new queens to replace her.
Queen bee pheromones prevent young bees from learning to sting until they leave the hive, which makes the hive safer.

However, the life of a honeybee is not hardwired. Bees are amazingly intelligent animals and learn a lot about their environment. In particular, forager bees learn color, odor, and position of nectar-rich flowers and use this information to optimize their harvest. This capacity has been used for many years to learn more about the basic mechanisms underlying appetitive (i.e., food-related) learning and memory, and the honeybee has become an important model animal to this end (5).

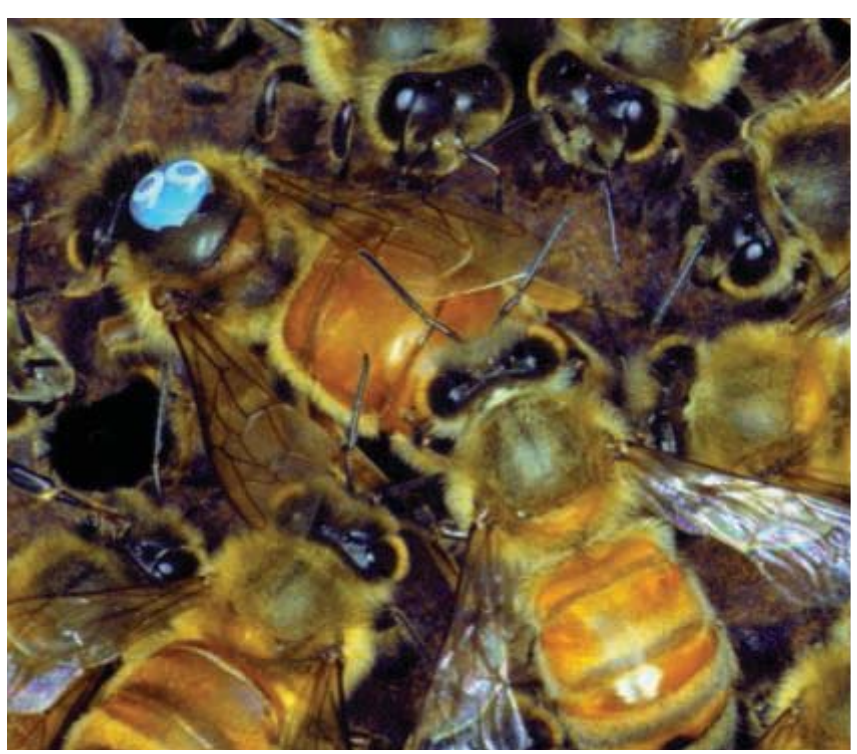

Chemical control. Nurse bees surround the queen (identified by the mark) and groom her. This behavior is important for distributing the queen's pheromone, QMP, within the entire hive. 
Recently, it has been shown that bees also learn to associate noxious stimuli, and an experimental paradigm has been developed whereby an odor can be associated with the sting reflex (6). In associative learning, a stimulus without meaning [a conditioned stimulus (CS) such as an odor] is associated with a reinforcer stimulus [an unconditioned stimulus (US) such as sweet nectar or a noxious electric shock]. The US elicits a response, for example, the extension of the tongue to lick the nectar or the extension of the sting for defense. After conditioning, the CS alone (e.g., the odor) will elicit the conditioned response. The neural substrates for an aversive US and for a positive US differ in bee brains, both in terms of the neurons involved and in terms of the neurotransmitter they use. The appetitive US channel uses octopamine as a transmitter (7), whereas the aversive US uses dopamine (6).

Is the learning capacity of a bee related to her developmental succession of tasks? Recent results have created an unprecedented link from molecules all the way to complex behavior, and some of the molecular effects of QMP are now understood. It turns out that QMP directly influences the chemistry of the brain in an age-dependent manner, contributing to developmental polyethism. One major component of QMP is homovanillyl alcohol (HVA), a substance with a striking chemical similarity to the biogenic amine dopamine, the neurotransmitter that mediates aversive learning. Indeed, QMP acts directly on the dopamine pathway: Levels of dopamine in the brain are reduced in young bees exposed to QMP, and this effect is amplified by a concurrent reduction in the levels of dopamine-sensitive receptors (8). Thus, exposure of a nurse bee to the queen's odor down-regulates the brain's dopamine networks and reduces activity levels.

What is the biological consequence of this down-regulation? Vergoz et al. now show that young bees exposed to QMP are not able to learn noxious stimuli. This defect is not a general learning deficit, because appetitive learning is not affected. Furthermore, the deficit is limited to young bees. Specifically, the authors show with aversive conditioning that young bees exposed to QMP cannot learn to extend their sting to an odor that has been presented together with an electric shock, whereas young bees not exposed to QMP are very good at this task. Aversive learning in older bees is also intact.

Thus, the presence of the queen, through her pheromone, influences the behavior, and indeed the brain pharmacology, of her hive. Young workers remain in the hive, are docile, and display less motor activity, whereas older guards and foragers leave the hive and become more motile and aggressive. Why would aversive learning be blocked in a young nurse bee? Within the hive, the sting reflex can only have negative effects. Thus, preventing nurse bees from developing aversive memories against the odors in the hive, which include the queen's own odor, makes the colony more secure. With increasing age, bees start to leave the colony, fly to distant foraging sites, and perform tasks outside the hive where they need to learn not only about sweet nectar but also about nasty dangers. It is useful, under these circumstances, that the effect of QMP to block aversive learning wanes. This is a wonderful example in which the effect of a releaser pheromone can be followed all the way to the neurons that are being modulated, and then to behavioral modifications.

Thus, honeybees differ substantially from the beings in Huxley's world, because individuals are not trapped within their castes for their entire life span. The manipulation of brain activity by the queen, modulating learning capacity in young bees to make them more docile, is a different view of parenthood. As the saying goes: When children are young, give them roots; when they grow, give them wings. The bee mother seems to have evolved exactly this strategy for her family.

\section{References}

1. A. Huxley, Brave New World (Doubleday Doran, Garden City, NY, 1932).

2. V. Vergoz, H. A. Schreurs, A. R. Mercer, Science 317,384 (2007).

3. B. Hölldobler, E. O. Wilson, The Ants (Belknap Press of Harvard Univ. Press, Cambridge, MA, 1990).

4. S. E. Fahrbach, G. E. Robinson, Dev. Neurosci. 18, 102 (1996).

5. R. Menzel, M. Giurfa, Trends Cogn. Sci. 5, 62 (2001)

6. V. Vergoz, E. Roussel, J. C. Sandoz, M. Giurfa, PLOS ONE 2, e288 (2007).

7. M. Hammer, Trends Neurosci. 20, 245 (1997)

8. K. T. Beggs et al., Proc. Natl. Acad. Sci. U.S.A. 104, 2460 (2007). 